\title{
Carcinoma de glándulas apocrinas con compromiso de vasos linfáticos de la dermis: reporte de dos casos
}

\author{
Carcinoma of the apocrine glands with involvement of lymphatic vessels \\ of the dermis: report of two cases \\ PC Jark ${ }^{\mathrm{a}}$, RR Huppes ${ }^{\mathrm{a}}$, OR Sierra ${ }^{\mathrm{a}^{*}}$, BP Maria ${ }^{\mathrm{a}}$, TMM Raposo ${ }^{\mathrm{a}}$, J Werner ${ }^{\mathrm{b}}$, CCV dos Reis ${ }^{\mathrm{a}}$, \\ CM Bueno ${ }^{a}$, R Laufer-Amorim ${ }^{c}, M_{\text {Tinucci-Costa }}{ }^{a}$, AB de Nardi ${ }^{\mathrm{a}}$ \\ aUniversidad Estadual Paulista, Campus de Jaboticabal, São Paulo, Brasil. \\ bLaboratorio Werner \& Werner, Curitiba, Brasil. \\ 'Universidad Estadual Paulista, Campus de Botucatu, São Paulo, Brasil.
}

\begin{abstract}
SUMMARY
Cutaneous inflammatory apocrine carcinoma is a non-common reported tumor which affects the skin causing multiple erosive lesions. The aim of this report is to describe two different cases of this atypical disease. Both cases presented with ulcerative and infiltrative lesions on cervical region. A surgical procedure was ruled out due to local infiltration and erosive condition. Case 1 was treated with bleomycin. Case 2 received just palliative treatment. In both cases the response was poor with survival times of 35 days and 15 days respectively. Clinical information about this disease is lacking on literature and more studies are warranted in order to describe better clinical presentation, treatment possibilities and prognostic.
\end{abstract}

Key words: carcinoma, apocrine gland, dog.

\section{RESUMEN}

El carcinoma apocrino inflamatorio cutáneo es un tumor poco reportado e infrecuente que afecta la piel causando lesiones múltiples erosivas. El objetivo de este reporte es describir dos diferentes casos que presentaron este infrecuente tumor. Ambos casos presentaron lesiones ulcerativas e infiltrativas en región cervical. Debido a la condición inflamatoria y erosiva de las lesiones, fue descartado un procedimiento quirúrgico. El caso 1 fue tratado con bleomicina, mientras que el caso 2 solo recibió tratamiento paliativo. En ambos casos la respuesta fue pobre con tiempos de sobrevida de 35 y 15 días respectivamente. En la literatura actualmente existe poca información acerca de esta enfermedad y es necesario que existan más estudios que logren describir mejor la presentación clínica, posibilidades de tratamiento y el pronóstico.

Palabras clave: carcinoma, glándula apocrina, canino.

\section{INTRODUCCIÓN}

El término apocrino denota el tipo de secreción glandular en el que la porción apical de la célula secretora se desprende junto con los productos secretorios acumulados dentro de ella (Souza y col 2009). Las glándulas sudoríparas apocrinas son glándulas que se localizan debajo de las glándulas sebáceas y se comunican con el interior del folículo piloso, arriba de la abertura del ducto sebáceo. Estas glándulas son más grandes y numerosas cerca de las uniones mucocutáneas, en los espacios interdigitales y en la superficie dorsal del cuello (Souza y col 2009).

Los carcinomas de glándulas apocrinas son tumores poco comunes en perros, localizados principalmente en miembros torácicos, siendo más común la presencia de nódulos únicos, sin embargo la presentación de dermatitis

Aceptado: 06.11.2014.

* Via de Acesso Prof. Paulo Donato Castellane s/n CEP: 14884-900, Jaboticabal, São Paulo, Brasil; osirra@ hotmail.com de tipo erosivo ulcerativo también es descrita en la literatura, siendo esta denominada como carcinoma inflamatorio cutáneo (Goldschmidt y Schofer 1998, Simko y col 2003, Baharak y col 2012, Pagnoncelli y col 2012).

Datos sobre el comportamiento clínico varían bastante entre los estudios, sin embargo se sugiere que generalmente este tipo de tumor se caracteriza por presentar infiltración local y en menor proporción por invadir vasos linfáticos de la dermis (Simko y col 2003). La tasa de metástasis de los carcinomas apocrinos cutáneos es baja en comparación a los carcinomas apocrinos de sacos anales. Existen pocos datos en la literatura al respecto de este tipo de tumor y de su respuesta al tratamiento empleado (Hauck 2013).

El objetivo del presente relato es describir dos casos de carcinoma apocrino en perros con invasión de los vasos linfáticos de la dermis, que presentaron comportamiento clínico agresivo y enfermedad progresiva después de la terapia empleada, incluso sin evidencia de metástasis a distancia, resaltando la necesidad de prudencia por parte de los oncólogos veterinarios al momento de establecer 
el pronóstico basado apenas en los datos actualmente disponibles acerca del comportamiento de esta neoplasia.

\section{MATERIAL Y MÉTODOS}

\section{REPORTE DE CASOS}

Dos caninos, hembras, sin raza definida, de 12 años de edad fueron atendidos por el departamento de oncología de la Universidad Estadual Paulista, Campus de Jaboticabal, con historia de lesión eritematosa e inflamatoria en piel. El caso 1 presentaba además drenaje de secreción serosa y presencia de material fibrinoso en algunos puntos en la región del miembro torácico izquierdo invadiendo la región cervical lateral, extremadamente adherido y con crecimiento rápido (figura 1). Según el propietario la lesión tenía 20 días de evolución. El caso 2 presentaba aspecto hemorrágico con drenaje de secreción serosa. En algunos lugares existía presencia de material necrótico en la región cervical lateral, además el tumor invadía la región cutánea de la escápula y se encontraba al igual que el caso 1 , fuertemente adherido y con crecimiento rápido. El tiempo de evolución reportado fue de 30 días. Ambos pacientes presentaban buen estado general y no había otras alteraciones clínicas. De manera rutinaria ante la sospecha de neoplasia fueron realizados exámenes de laboratorio (hemograma y bioquímica sérica) encontrándose solo una leve leucocitosis con neutrofilia en el hemograma
Cuadro 1. Hemograma canino del caso 1.

Canine hemogram of case number 1 .

\begin{tabular}{lcc}
\hline Parámetro & Rango normal & Resultado \\
\hline Glóbulos rojos & $5,5-8,5 \times 10^{12} / \mathrm{L}$ & 6,3 \\
Hematocrito & $37-52 \%$ & 38,1 \\
Hemoglobina & $12-18 \mathrm{~g} / \mathrm{dl}$ & 12,7 \\
Glóbulos blancos & $6-14 \times 10^{9} / \mathrm{L}$ & 18,1 \\
Neutrófilos segmentados & $56-78 \% ; 4-10,6 \times 10^{9} / \mathrm{L}$ & $79 \% ; 14,2$ \\
Linfocitos & $13-30 \% ; 0,9-5,1 \times 10^{9} / \mathrm{L}$ & $18 \% ; 3,2$ \\
Monocitos & $3-10 \% ; 0,2-1,5 \times 10^{9} / \mathrm{L}$ & $2 \% 0,36$ \\
Bandas & $0-3 \% ; 0-0,3 \times \times 10^{9} / \mathrm{L}$ & $1 \% 0,18$ \\
Plaquetas & $200.000-400.000$ & 290.000 \\
\hline
\end{tabular}

del caso 1 (cuadro 1). En los demás exámenes no fueron encontradas alteraciones. Igualmente fueron realizadas radiografías torácicas y ecografía abdominal en búsqueda de metástasis, y radiografía de miembro afectado para evaluar un posible compromiso óseo. Fue también realizada citología de la lesión y posteriormente se realizó biopsia incisional para confirmar la sospecha de neoplasia epitelial arrojada por la citología.

Ante la evidencia de tumor apocrino en piel, se realizó palpación de glándulas de saco anal para descartar una posible metástasis de carcinoma apocrino de saco anal a piel. Posteriormente frente a la imposibilidad de resección quirúrgica en ambos casos debido a la extensión de la masa,

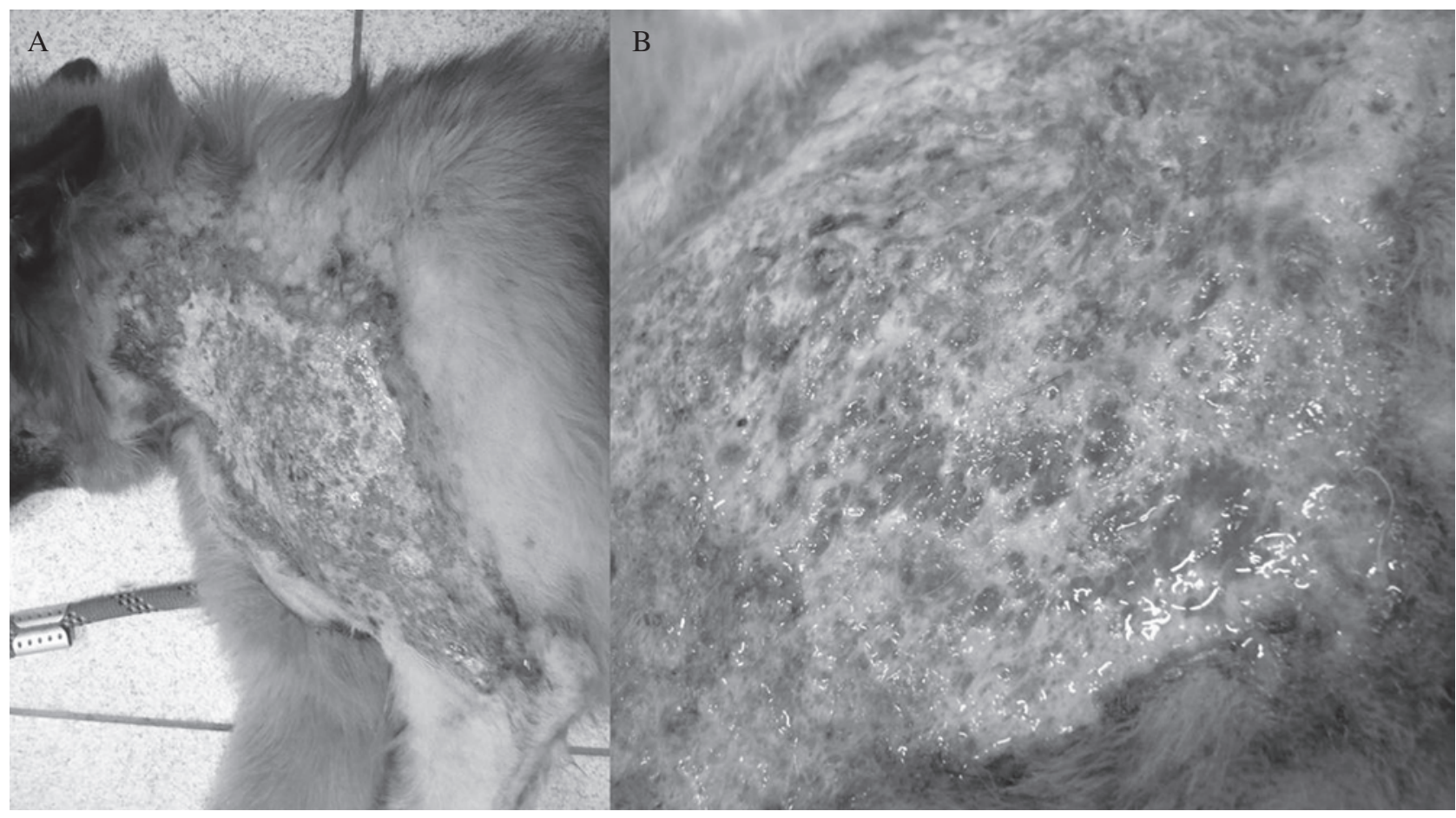

Figura 1. A. Canino, sin raza definida, hembra, 12 años, con lesión inflamatoria en miembro torácico izquierdo, invadiendo región cervical. B. Lesión inflamatoria, ulcerada y erosiva diagnosticada como carcinoma apocrino con invasión de vasos linfáticos de la dermis.

A. A 12 year old, female, mixed breed dog presenting with inflammatory lesion on left front limb, involving cervical area. B. Inflammatory, ulcerated and erosive lesion diagnosed as carcinoma of the apocrine glands with involvement of the lymphatic vessels of the dermis. 
fue propuesto tratamiento paliativo mediante limpieza diaria de la herida, firocoxib (5 mg/kg SID), cefalexina (30 mg/kg BID), clorhidrato de tramadol (3 mg/kg TID). Para el caso 1 fue realizada quimioterapia antineoplásica usando bleomicina (15 UI/m2) en inyección subcutánea 1 vez por semana. Luego de dos sesiones de este protocolo el paciente continuó presentando enfermedad progresiva junto con cuadro de anorexia y pérdida de peso progresiva. El paciente murió 35 días después del diagnóstico. Para el caso 2 no fue autorizada la quimioterapia por parte del propietario, el paciente también continuó con enfermedad progresiva presentando pérdida de peso, vómito y anorexia concomitantes. Después de 15 días del diagnóstico el paciente murió. No fue posible realizar necropsia en ninguno de los casos por no ser autorizada por parte de los propietarios.

\section{RESULTADOS Y DISCUSIÓN}

El resultado de la citología fue sugerente de neoplasia de origen epitelial, más a fondo el estudio histopatológico reveló una proliferación neoplásica infiltrativa, poco delimitada y sin revestimiento de cápsula fibrosa que afectaba toda la dermis y el panículo adiposo. Las células tumorales eran poliédricas, grandes y se propagaban formando pequeños grupos compactos y estructuras glandulares ductales irregulares. Fue también observado anisocariosis, anisocitosis, atipia nuclear, nucléolos evidentes y desmoplasia intensa, además de áreas de necrosis tumoral. El índice mitótico fue menor a 1 f.m./40x, con mitosis aberrantes. Adicionalmente fue observada la presencia de émbolos neoplásicos en los vasos linfáticos de la dermis, semejantes a los observados en carcinomas mamarios inflamatorios.
El diagnóstico fue de carcinoma de glándulas apocrinas con invasión de vasos linfáticos (figura 2).

Los carcinomas de glándulas apocrinas son tumores poco frecuentes en perros y la literatura disponible respecto de esta neoplasia aborda principalmente la morfología celular describiendo adicionalmente otros hallazgos histopatológicos como infiltración dérmica y subcutánea con reacción dermoplástica variable, núcleos con gran cantidad de cromatina, nucléolos prominentes y fagocitosis neutrofílica (Goldschmidt y Schofer 1998). Existiendo por tanto pocos datos sobre el comportamiento clínico, formas de tratamiento y pronóstico de este tumor (Simko y col 2003).

Algunos autores sugieren mayor prevalencia en animales entre 8 y 12 años de edad, sin predilección sexual aparente (Kalaher y col 1990, Simko y col 2003, Goldschmidt y Hendrick 2007). La edad citada en la literatura corrobora con la encontrada en los animales del presente reporte, ya que ambos presentaban 12 años de edad.

Los datos disponibles acerca del comportamiento clínico del carcinoma apocrino muestran informaciones controversiales. Sin embargo algunos autores citan que los carcinomas de glándulas apocrinas presentan potencial de metástasis a distancia e invasión local. Kalaher y col (1990) evaluaron 44 caninos con carcinoma de glándulas apocrinas. Ningún animal presentó metástasis a distancia, aunque nueve animales presentaron invasión de los vasos linfáticos de la dermis como lo descrito en los pacientes de este reporte.

Simko y col (2003) realizaron un estudio retrospectivo con 44 carcinomas de glándulas apocrinas y apenas un caso presentó metástasis a distancia. En relación con el

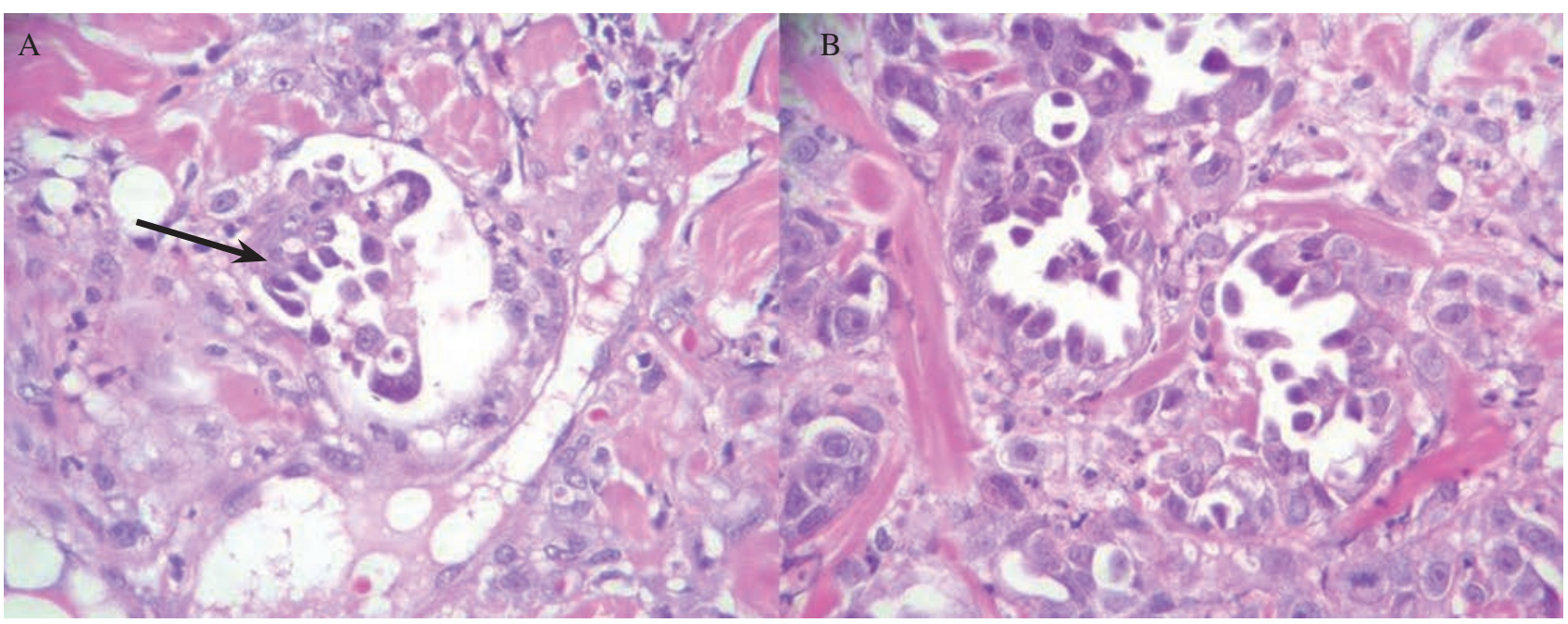

Figura 2. Fotomicrografía de piel con diagnóstico de adenocarcinoma apocrino. A. Nótese los vasos linfáticos de la dermis con contenido de émbolos neoplásicos (flecha). (H.E. Aumento de 400x). B. Nótese la proliferación desordenada con formación de bloques compactos y estructuras glandulares irregulares. La imagen muestra también anisocariosis, atipia nuclear y nucléolos evidentes y atípicos (H.E. Aumento de 400x).

Skin photomicrography diagnosed with carcinoma of apocrine gland. A. Note the lymphatic vessels of the dermis with neoplastic emboli (arrow) (H.E. 400x). B. Note the disorganized proliferation with formation of compact clusters and irregular gland structures. The image also shows anisokaryosis, nuclear atypia, evident and atypical nucleoli (H.E. 400x). 
tiempo de sobrevida de estos pacientes, de los 25 animales que presentaban datos de seguimiento apenas un animal fue sometido a eutanasia por causa del tumor, siete caninos murieron como consecuencia de enfermedades no relacionadas con el tumor (ninguno de estos pacientes fue sometido a necropsia para confirmación de la causa de muerte) y 17 animales estaban vivos y sin señales de recurrencia de la enfermedad al término del estudio. La sobrevida media en este estudio fue de 30 meses después de la cirugía, sin embargo el tamaño medio de las lesiones fue de $2 \mathrm{~cm}$ de diámetro y $89 \%$ eran masas solitarias, lo que puede haber influenciado para un pronóstico más favorable en relación con los pacientes presentados en este relato, puesto que las lesiones descritas son de tipo infiltrativo, no nodular y con invasión de vasos linfáticos de la dermis. Posiblemente existen dos formas de presentación de los carcinomas apocrinos cutáneos; la nodular que presenta posibilidad de remoción quirúrgica y la forma más infiltrativa difusa como la presentada en este caso que denota un difícil tratamiento.

Baharak y col (2012) describieron un caso de carcinoma de glándulas apocrinas con recurrencia local y metástasis a distancia nueve meses después de remoción del tumor primario de 5,5 x $3,5 \mathrm{~cm}$, pedunculado, cerca de la región auricular izquierda. A pesar de que la metástasis no haya sido detectada en los exámenes de imagen en los dos casos relatados, esta no puede ser descartada, ya que los animales no fueron sometidos a examen de necropsia.

Pagnoncelli y col (2012) describieron un caso de carcinoma de glándulas apocrinas solitario de crecimiento lento que después de un año de evolución presentó evolución progresiva y rápida, llegando a medir $16 \mathrm{~cm}$ de diámetro, con presencia de ulceración significativa y desarrollo de nuevos nódulos cutáneos una semana después de la resección quirúrgica de tumor primario, con evolución desfavorable del caso y muerte cinco meses después de la intervención quirúrgica.

La resección quirúrgica parece ser el tratamiento de elección para este tipo de tumor y los datos sobre el tratamiento quimioterapéutico son escasos en la literatura. No fueron encontrados estudios sobre la utilización de inhibidores de COX-2 en el tratamiento de carcinomas de glándulas apocrinas, sin embargo la utilización de estos medicamentos en los casos relatados fue principalmente para el control de la inflamación local, aspecto de las lesiones y extrapolación del uso de esta clase de fármacos en otros tipos de carcinoma cutáneos que presentaron expresión de COX-2 (Almeida y col 2001).

En relación con el pronóstico de la enfermedad, los resultados son controvertidos, aunque parezcan relacionados principalmente con el tamaño, número de lesiones, características microscópicas, grado de infiltración local y presencia de metástasis a distancia (Kalaher y col 1990, Simko y col 2003). En este caso el curso agresivo principalmente relacionado con la invasión local y deterioro del cuadro clínico de los pacientes sin evidencia de metástasis, hace pensar que existe un tipo de carcinoma de glándulas apocrinas con características de infiltración difusa y con comportamiento clínico también agresivo.

\section{REFERENCIAS}

Almeida EMP, C Piché, J Sirois, M Doré. 2001. Expression of cyclooxygenase- 2 in naturally occurring squamous cell carcinomas in dogs. J Histochem Cytochem 49, 867-875.

Baharak A, K Reza, D Shahriar, A Omid, V Daruoosh, A Nasrin. 2012. Metastatic apocrine sweat gland adenocarcinoma in a terrier dog. Asian Pac J Trop Biomed 3, 670-672.

Goldschmidt MH, FS Schofer. 1998. Apocrine gland tumors. In: Goldschmidt MH, Schofer FS (eds). Skin tumors of the dog and cat. Butterworth-Heinemann, Woburn, USA, Pp 80-95.

Goldschmidt MH, MJ Hendrick. 2007. Tumors of the skin and soft tissues. In: Meuten DJ (ed). Tumors in domestic animals. Iowa State University Press, Ames, USA, Pp 72-73.

Hauck ML. 2013. Tumors of the skin and subcutaneous tissues. In: Withrow SJ, Vail DM, Page RL (ed). Withrow \& MacEwen's Small Animal Clinical Oncology. $5^{\text {th }}$ ed. Saunders Elsevier, Missouri, USA, Pp 305-320.

Kalaher KM, WI Anderson, DW Scott. 1990. Neoplasms of the apocrine sweat glands in 44 dogs and 10 cats. Vet Rec 127, 400-403.

Pagnoncelli M, DB Martins, RT Franca, STA Lopes, RA Zanette, CM Mazzanti. 2012. Fine-needle aspiration cytology of the canine apocrine sweat gland carcinoma. Comp Clin Path 21, 627-629.

Simko E, BP Wilcock, JA Yager. 2003. A retrospective study of 44 canine apocrine sweat gland adenocarcinomas. Can Vet J 44, 38-42.

Souza TM, RA Fighera, GD Kommers, CSL Barros. 2009. Aspectos histológicos da pele de cães e gatos como ferramenta para dermatopatologia. Pesquisa Vet Brasileira 29, 177-190. 